
\title{
25 Research Square \\ IL-31, IL-32 and IL-33 may Serve as Diagnosis Biomarkers in Gastric Cancer
}

Shisan Bao ( $\sim$ bob.bao@sydney.edu.au )

University of Sydney

Qinghua Liu

Xuzhou Medical University

Jiwei Zhang

Songjiang Hospital, Shanghai Jiaotong University School of Medicine

\section{Lei Xia}

Xuzhou Medical University

\section{Steven Wise}

University of Sydney

\section{Brett Hambly}

University of Sydney

\section{Kun Tao}

Tongren Hospital, Shanghai Jiaotong University School of Medicine

\section{Research Article}

Keywords: diagnosis and therapy, gastric cancer, immune cell interactions

Posted Date: December 21st, 2020

DOI: https://doi.org/10.21203/rs.3.rs-128062/v1

License: (c) (i) This work is licensed under a Creative Commons Attribution 4.0 International License.

Read Full License 


\section{Abstract}

Background: To determine whether IL-31, IL-32 and IL-33 can be used as biomarkers for the detection of gastric cancer (GC), via evaluating the correlations between IL-31, IL-32 and IL-33 expression and clinicopathological parameters of GC patients.

Methods: Tissue array ( $\mathrm{n}=180)$ gastric specimens were utilised. IL-31, IL-32 and IL-33 in GC and non-GC tissues were detected immunohistochemically. The correlations between IL-31, IL-32 and IL-33 in GC and severity of clinicopathological parameters were evaluated. Survival curves were plotted using the KaplanMeier/Cox regression. IL-31, IL-32 and IL-33 in circulation were detected by ELISA.

Results: IL-31, IL-32 and IL-33 were all lower in GC than that in adjacent non-GC gastric tissue ( $p$ all $<0.05)$. IL-33 in peripheral blood of GC patients was significantly lower than that of healthy individuals $(1.50 \pm 1.11 \mathrm{vs} 9.61 \pm 8.00 \mathrm{ng} / \mathrm{ml})(p<0.05)$. Decreased IL-31, IL-32 and IL-33 in GC were observed in younger patients ( $<60$ years), and IL-32 and IL-33 were lower in female patients ( $p$ all $<0.05$ ). Higher IL-32 correlated with longer survival in two GC subgroups: T4 invasion depth and TNM I-II stage.

Univariate/multivariate analysis revealed that IL-32 is an independent prognostic factor for GC within the T4 stage subgroup. Circulation IL-33 was significantly lower in GC patients than healthy people $(p<0.05)$.

Conclusions. Our findings provide new insights into the roles of IL-31, IL-32 and IL-33 in carcinogenesis of $\mathrm{GC}$ and demonstrate their relative usefulness as prognostic markers for GC. The underlying mechanism of IL-31, IL-32 and IL-33 in GC is further discussed.

\section{Introduction}

Gastric cancer (GC) is one of the most common malignancies in China with a high morbidity $(\sim 24 \%)$ and mortality $(\sim 17 \%)[1]$, and is ranked third amongst malignant tumours[2]. Despite the more widespread use of recent diagnostic advances, including endoscopic examination, many of GC patients are diagnosed with advanced stage disease, resulting in a poor five year survival rate $(<20 \%)$, emphasising the need for development of a reliable biomarker(s)[3]. Therefore, it is critically important to develop reliable biomarker(s) with high specificity and sensitivity, to improve early diagnosis for more successful outcomes for GC patients. There is a concept that the development of cancer is determined partially by

The competent body immunity is critically important in inhibiting the development of malignancy[4], but it remains to be explored the precise underlying mechanism how host defence in the oncogenesis of gastric cancer [5]. It is accepted that the role of proinflammatory or anti-inflammatory response during the development of malignancy, which may either stimulate or inhibit the growth of a cancer [6]. The discovery of the immune checkpoint molecules PD-1 and CTLA-4 is elegantly demonstrated [7] via inhibiting anti-cancer immunity during oncogenesis [8]. In addition, it has also been studied that carcinogenesis in the gastrointestinal system [9], including subtypes of colorectal cancer, as well as the genetic mutations [9]. Furthermore, cytokine expression has been clearly associated with the modulation of CRC[6, 10]. 
Helicobacter pylori, a spiral Gram-negative rod that infects and colonizes the human stomach in $50 \%$ of the human population, is a definite human oncogenic agent[11]. In addition, it has been suggested that $H$. pylori contributes to $>60 \%$ of all stomach cancers, although the precise underlying mechanism remains to be explored[12]. However, it has been well illustrated by the Nobel laureate Barry Marshal that chronic gastric ulceration is caused by $\mathrm{H}$. pylori infection, which can be eliminated by cocktails of antibiotics[13]. Thus, it is reasonable to speculate that host immunity plays a critical role during the development of gastric cancer.

The cell-mediated immune response is extremely important in defence against tumour development, since compromised host immunity is known to contribute to the establishment, proliferation and metastasis of malignant tumours[14]. Although high host inflammatory status has been reported in the tumour microenvironment, an incompetent inflammatory/immune response will lead to tumour progression[15].

Interleukin 31 (IL-31), an immunoregulatory cytokine secreted mainly by activated Th2 cells, plays a major role in the process of chronic inflammation[16]. However, the involvement of this interleukin in the pathogenesis of cancer is unclear. Recent studies have shown that malignant Tcells produce IL-31, with an associated increase in serum levels of IL-31[17]. Additionally, in the advanced stages of cutaneous T cell lymphoma patients, improved pruritus correlates with lower levels of IL-31[18].

IL-32, a proinflammatory cytokine, is highly produced in several autoimmune diseases, e.g. rheumatoid arthritis, inflammatory bowel disease and atopic dermatitis[19, 20]. However, by contrast with autoimmune and inflammatory diseases, the role of IL-32 appears to fifer amongst different forms of cancer, e.g. IL-32 exhibits anti-tumour effects in human colon cancer and leukaemia[21, 22], however, it promotes tumorigenesis in human pancreatic cancers[23]. The role of IL-32 in GC is controversial, i.e. one study found that IL-32 expression is elevated in GC compared with normal stomach[24], while another study reported that there is no significant difference between GC and normal stomach[25]. The precise role of IL-32 in tumorigenesis of GC and other malignancies remains to be fully explored.

IL-33, a member of the IL-1 family, regulates innate and adaptive immunity as a potent inducer of proinflammatory cytokines. The involvement of IL-33 in non-small cell lung cancer is controversial, i.e. high IL-33 has been found to be of diagnostic and prognostic value[26], but another group reports no significant associations[27]. The possible role IL-33 in GC remains to be explored.

In our study we aim to identify the expressions of IL-31, IL-32 and IL-33 in GC and assess their intercorrelation and clinical significance. Our data may provide useful information for both our basic understanding of tumour immunology and/or therapeutic targets for GC patients.

\section{Methods}

\section{Patients and samples}


GC tissues and adjacent histologically normal gastric tissues (control) were obtained from $180 \mathrm{GC}$ patients undergoing gastrectomy in the Affiliated Hospital, Xuzhou Medical University, China, between 2008 and 2010. These GC patients were comprised of 140 males and 40 females, aged from 23 to 85 years old (Table 1). No chemotherapy was administered to these patients prior to gastrectomy.

All patients have complete clinical information. Among them, 77 had follow-up until their death or until their most recent contact (May, 2015). At the time of the most recent contact, 14 of 77 were still alive, whereas 42 were dead and the other 21 were lost contacts during the following-up. Among the14 surviving patients, the longest survival period was 76 months. This study was approved by the Human Ethical Committee, the Institutional Review Boards of Affiliated Hospital of Xuzhou Medical University, and written informed consents were obtained.

\section{Immunohistochemistry}

Sections $(5 \mu \mathrm{m})$ from tissue microarray blocks were labelled with three antibodies, as described previously[28]. The antibodies were: rabbit anti-IL-31 polyclonal antibody, (22859-1-AP, Proteintech, China), rabbit anti-IL-32 polyclonal antibody, (11079-1-AP, Proteintech, China) and rabbit anti-IL-33 polyclonal antibody, (12372-1-AP, Proteintech, China). The dilution for all three antibodies was 1:100. HRP-conjugated secondary antibody (12127A07, Beijing Sequoia Jinqiao Biological Technology Co., Ltd.) was used. The specific target(s) was visualized with a DAB detection kit (Beijing Sequoia Jinqiao Biological Technology Co., Ltd.) and counterstained with hematoxylin.

Photomicrographs from each of the tissue arrays were taken with a fixed exposure time and colour balance to ensure consistency. IL-31, IL-32 and IL-33 productions were quantified using ImagePro Plus9.1, (Media Cybernetic, Silver Spring, MD), as described previously[29].

\section{ELISA for IL-31, IL-32 and IL-33}

To determine if there was a correlation between GC and circulating IL-31, IL-32 and IL-33, we had enrolled prospectively $10 \mathrm{GC}$ patients prior to preoperative chemotherapy in the Affiliated Hospital, Xuzhou Medical University, China. Ten healthy non-cancer persons presenting for a routine health check-up were collected as controls in our study. Consents were obtained from both GC patients and healthy control. The circulating cytokine study has also been approved by the Human Ethical Committee, the Institutional Review Boards of Affiliated Hospital of Xuzhou Medical University. Plasma samples were collected from subjects and were stored at $-80^{\circ} \mathrm{C}$ until analysis. The concentrations of IL-31, IL-32 and IL-33 were determined using an ELISA instrument (Bio-Rad 550, US) at $450 \mathrm{~nm}$, followed the manufacturers' instructions for human IL-31 (KGEHC141, KeyGEN BioTECH, Nanjing, China), IL-32 (SEB802Hu, CloudClone Corp, Wuhan, China) and IL-33 (KGEHC151, KeyGEN BioTECH, Nanjing, China). All samples were tested in duplicate. 


\section{Statistical analysis}

GraphPad Prism 6.0 and SPSS 16.0 statistical software packages were used for the statistical analysis of the results of immunohistochemistry. Comparison between two groups was performed via MannWhitney $\mathrm{U}$ test. Comparisons among multi-groups were performed via Kruskal-Wallis test. Low and high cutoff values for cytokine expression were defined by ROC curve analysis. Survival curves were plotted by the Kaplan-Meier method and compared by the log-rank test. Cox's proportional hazards model was used to identify the prognostic factors that influenced survival. $p<0.05$ was considered statistically significant [30].

\section{Results}

\section{IL-31, IL-32 and IL-33 local expression of in GC tissue and in peripheral blood of GC patients}

The expression levels of IL-31 (Fig. 1A, B), IL-32 (Fig. 1D, E) and IL-33 (Fig. 1G, H) in GC tissue were investigated, using immunohistochemistry. The densities of IL-31 (Fig. 1C), IL-32 (Fig. 1F) and IL-33 (Fig. 1I) were presented as box plots, including medians and 25th and 75th percentiles. IL-31, IL-32 or IL33 were decreased by $9.4 \%, 28.2 \%$ or $27.5 \%$, respectively, in GC compared to histologically normal adjacent gastric tissues $(\mathrm{p}<0.05)$.

There was no significant different IL-31 (Fig. 1J) or 32 (Fig. 1K) in the peripheral blood between GC patients and heathy control. However the mean value for IL-33 levels in peripheral blood of GC patients was $1.50 \pm 1.11 \mathrm{ng} / \mathrm{ml}$, which was significantly lower than that of healthy individuals $(9.61 \pm 8.00 \mathrm{ng} / \mathrm{ml})$ $(p<0.05)$ (Fig. 1L).

\section{Correlation between IL-31, IL-32 and IL-33 in GC and clinicopathological parameters}

Associations between clinicopathological parameters and IL-31, IL-32 and IL-33 expression are listed in table 1, Figs. 2, 3 and supplementary Figs. 1, 2. IL-31 (A-D), IL-32 (E-H) and IL-33 (I-L) were all associated with the age of GC patients (Fig. 2). There was significantly lower expression of IL-31, IL-32 and IL-33 in the group of GC patients aged $\leq 60$ years compared to the patients aged $>60$ (all $p<0.05$ ). Significantly lower IL-32 (A-D) and IL-33 (E-H) expression was also observed in female GC patients compared to male GC patients (both $p$ < 0.05) (Fig. 3). However, no significant differences were observed in IL-31 with gender of GC (supplementary Fig. 2). Additionally, there were no correlations observed among IL-31, IL-32 and IL33 and other parameters, such as tumour size, lymph node metastasis, tumour differentiation, tumour invasion depth (supplementary Fig. 1) and TNM stage (supplementary Fig. 2) of GC. 


\section{Prognostic cytokines for overall survival of GC patients}

Correlation of decreased IL-31, IL-32 and IL-33 with overall survival of GC patients

To evaluate whether decreased IL-31, IL-32 and IL-33 correlated with survival of GC patients, low and high cut-off points for IL-31 (A), IL-32 (B) and IL-33 (C) were defined by ROC curve analysis (Fig. 4). KaplanMeier survival curves were constructed to compare high and low expression of IL-31 (D), IL-32 (E) and IL$33(F)$ in the survival of GC patients. Our data revealed that there were no correlations of IL-31, IL-32 and IL-33 with the prognosis of GC patients (Fig. 4). However, Kaplan-Meier analysis was applied to further compare overall survival according to IL-31 (G), IL-32 (H) and IL-33 (I) in different subgroups of GC. Figure 4 shows decreased IL-32 staining correlated with significantly worse survival of patients in the TNM I-II tumour stage subgroup $(p=0.006)(K)$ and tumour invasion depth T4 subgroup $(p=0.004)$. There was no significant differences found in the other subtypes of IL-31, IL-32 and IL-33 (supplementary Figs. 3-5). Furthermore, there was no significant differences found in the combination of IL-31, IL-32 and IL-33 for the prognosis of GC patients (supplementary Fig. 6).

\section{Further analysis of the correlation of IL-32 with overall survival in subgroups of GC patients}

Univariate and multivariate Cox regression analysis was used to examine whether IL-32 was an independent prognostic marker for subgroups of GC patients, including IL-32 level of expression, age, gender, tumour differentiation, lymph node invasion, tumour size, the depth of tumour invasion and TNM stage.

Data from patients within the T4 stage subgroup, analysed by univariate analysis (Table 2) exhibited a correlation between the survival of GC patients and IL-32 expression and TNM stage, respectively. In multivariate analysis, IL-32 and TNM stage remained as significant independent prognostic factors for survival of GC patients.

Furthermore, decreased survival of GC patients within the TNM I-II stage subgroup was found to correlate with lymph node metastasis and tumour size on univariate analysis, but not on multivariate analysis. However, both univariate and multivariate analysis revealed that no significant correlations between decreased IL-32 and survival of GC patients within the TNM I-II stage subgroup of GC patients (Table 3).

\section{Discussion}

The current study has demonstrated that the levels of expression of IL-31, IL-32 and IL-33 are all decreased within GC tissue compared to adjacent normal control gastric tissue and that these reductions in expression are higher in younger patients below the age of 60 years. Additionally, in the case of IL-32 and IL-33, expression was found to be lower in females compared to males. However, the level of expression of all three interleukins amongst all the GC patients as a group did not correlate with a 
survival benefit, although subgroup analysis did reveal a survival benefit associated with higher levels of expression of IL-32 within the T4 stage and the TNM I-II stage subgroups.

This study found decreased IL-31 expression in GC patients, particularly in younger patients. Our data are consistent with other studies that have shown that younger patients are more likely to have more poorly differentiated tumours compared to older patients with GC, suggesting that younger GC patients have more malignant types of GC[31]. The activity of IL-31 is mediated through the IL-31 receptor A (IL-31RA) and the Oncostatin M Receptor (OSMR)[32,33]. The two different isoforms of the IL-31RA consist of either long (745 residues) or short (560 residues) isoforms which may induce contrary functions[34]. Proliferation of follicular lymphoma is enhance via the long signalling IL 31RA isoform; whereas germinal centre-derived B-cell malignancy is inhibited via the short IL-31RA isoform[35]. There is no direct evidence that identifies which isoform/s of IL31RA are activated in GC via the IL-31 signalling pathway. However, our data are consistent with the hypothesis that IL-31 may mediate an anti-cancer role in GC through the short IL-31RA isoform.

The data from the current study also shows similar levels of expression of IL-31 and IL-33 in GC, with decreased IL-33 in both younger GC patients and in female GC patients. Our data are consistent with a report that female sex is a significant factor for predicting a higher likelihood of lymph node metastasis in mucosa-confined, poorly differentiated GC[36], consistent with the hypothesis that part of the explanation for GC in females being more severe than in males may be the lower levels of IL-33 found in females compared to males. IL-33 is a multifunctional cytokine, that can bind to IL-33 receptor (ST2), to regulate the immune system through activating Th1 cells, Th2 cells, CD8 ${ }^{+} \mathrm{T}$ cells and NK cells[37, 38]. There are two kinds of ST2: the transmembrane form ST2L, that when bound to IL-33 is able to activate target cells[39] and the soluble, secreted form of ST2 (SST2) that acts as a decoy receptor and negatively regulates IL-33 signalling[40]. The possible role of IL-33 in carcinogenesis has been demonstrated via transgenic over-expression of IL-33 in IL-33 transgenic mouse metastasis model, which demonstrated inhibition of the growth and metastasis of B16 melanoma and Lewis lung carcinoma cells, via activating $\mathrm{CD}^{+} \mathrm{T}$ cells and NK cells[41]. These data may be useful for future therapeutic design, utilising the anticancer role of IL-33 in GC.

However, the role of IL-32 in malignancy is controversial, e.g., IL-32 has been shown to enhance proliferation of human pancreatic cancer [23], but is able to suppress human colon cancer and leukaemia[21, 22]. In the current study, our results show that the expression of IL-32 was decreased in both younger patients and in female patients with GC, where both younger patients and female patients experience more severe forms of GC. These data suggest that IL-32 may mediate host defence against the development of GC. Furthermore, we found that high IL-32 expression correlated with longer survival of GC patients, within the T4 stage and TNM I-II stages subgroups and that IL-32 is an independent prognostic factor for survival within the T4 stage subgroup with GC. Interestingly, the IL-32-positive rate in GC $(12 \%)$ has been reported to be much lower than the rate in oesophageal squamous cell carcinoma (60\%), but no comparison to noncancerous tissues has been undertaken[42, 43]. 
Thus, we propose an hypothesis for the possible mechanism of IL-32 involvement in carcinogenesis as follows: Because IL 32 contributes to the host defence system via enhancing differentiation of monocytes into macrophage[44], decreased IL-32 in GC tissue, seen particularly amongst the younger or female patients, may compromise host innate immunity, and subsequently contribute to uncontrolled development of cancer. Notably, macrophages are classified as either classical M1 cells, that promote the inflammatory response against microorganism invasion; or as M2 macrophages that regulate host immunity[45]. It remains to be clarified whether tumourassociated macrophages are derived from one subset or the other, which either promote the development of cancer or suppress cancer growth, which is perhaps dependent on the tumour micro environment[46]. For example, IL-32 can induce cell death in thyroid cancer cells through the induction of IL-8 and caspase-8[47], subsequently up-regulating proinflammatory response.

IL-32 may also be able to inhibit tumour growth indirectly, hence it may be efficacious as a clinical anticancer therapy[48]. For example, the application of siRNA to inhibit IL-32 enhances angiogenesis in HUVECs[49] via up-regulation of VEGF and PDGF. Our current findings have shown an inverse correlation between IL 32 and the development of GC, also suggesting that IL-32 may inhibit the development of cancer directly and indirectly, which will be further investigated in future experiments.

Unfortunately, no correlation with survival of GC patients was observed among any combination of IL-31, IL-32 and IL-33 expression, a similar result that we have reported previously for the relationship between IL-34 in GC[30]. Such a discrepancy among IL-31, 32, 33 and 34 may be due to different receptors and/or signalling pathways, which will be clarified in the conditioning knockout mice in future studies.

\section{Conclusions}

In summary, our data demonstrate that IL-31, IL-32 and IL-33 expression in GC were all decreased, which was correlated with young age of the GC patients. IL-32 and IL-33 were also correlated with the gender of the GC patients. Decreased IL-32 was correlated with poorer survival of GC patients within the T4 stage and TNMI II stage subgroups. Downregulation of IL-32 was an independent prognostic factor for T4 GC patients. Finally, low IL-33 in peripheral blood may be considered as a biomarker for the diagnosis of GC. However, further studies are required to investigate the mechanism of the role of these interleukins in GC.

\section{Declarations}

\section{Ethics approval and consent to participate:}

This study was approved by the Human Ethical Committee, the Institutional Review Boards of Affiliated Hospital of Xuzhou Medical University in accordance with the Declaration of Helsinki, and written informed consents were obtained.

\section{Consent for publication:}




\section{Availability of data and materials:}

The datasets used and/or analysed during the current study available on reasonable request

\section{Competing interests:}

The authors declare no conflicts of interest

\section{Funding:}

This study was supported by grants from National Natural Science Foundation of China (NO. 81502030). SJTU research grant 2019, Sydney University. Liu was a recipient of a Travel Fellowship, Minster of Education, Jiangsu Province, China.

\section{Authors' contributions:}

$\mathrm{QL}$, collected the samples, performed histopathology, immunohistochemistry, data analysis and wrote the paper; JZ, collected sample, data analysis and wrote the paper: LX, performed ELISA and data analysis; $S G W$, intellectual input; $B D H$, revised the manuscript, and intellectual input; $K T$, intellectual input, revised the paper, SB, designed the experiment, revised the manuscript.

\section{Acknowledgments:}

We acknowledge the General staffs from Department of Pathology, Xuzhou Medical University for their support.

\section{References}

1. Zheng R, Zeng $\mathrm{H}$, Zhang S, Chen W: Estimates of cancer incidence and mortality in China, 2013. Chin J Cancer 2017, 36(1):66.

2. Fock KM: Review article: the epidemiology and prevention of gastric cancer. Aliment Pharmacol Ther 2014, 40(3):250-260.

3. Yoon H, Kim N: Diagnosis and management of high risk group for gastric cancer. Gut Liver 2015, 9(1):5-17.

4. Cali B, Molon B, Viola A: Tuning cancer fate: the unremitting role of host immunity. Open Bio/ 2017, 7(4). 
5. Ponz de Leon M, Benatti P, Borghi F, Pedroni M, Scarselli A, Di Gregorio C, Losi L, Viel A, Genuardi M, Abbati $\mathrm{G}$ et al: Aetiology of colorectal cancer and relevance of monogenic inheritance. Gut 2004, 53(1):115-122.

6. Mager LF, Wasmer MH, Rau TT, Krebs P: Cytokine-Induced Modulation of Colorectal Cancer. Front Oncol 2016, 6:96.

7. Zang X: 2018 Nobel Prize in medicine awarded to cancer immunotherapy: Immune checkpoint blockade - A personal account. Genes Dis 2018, 5(4):302-303.

8. Siegel R, Desantis C, Jemal A: Colorectal cancer statistics, 2014. CA Cancer J Clin 2014, 64(2):104117.

9. De Robertis M, Poeta ML, Signori E, Fazio VM: Current understanding and clinical utility of miRNAs regulation of colon cancer stem cells. Semin Cancer Biol 2018, 53:232-247.

10. Wei SC, Duffy CR, Allison JP: Fundamental Mechanisms of Immune Checkpoint Blockade Therapy. Cancer Discov 2018, 8(9):1069-1086.

11. IARC working group on the evaluation of carcinogenic risks to humans: some industrial chemicals. Lyon, 15-22 February 1994. IARC Monogr Eval Carcinog Risks Hum 1994, 60:1-560.

12. Parkin DM: The global health burden of infection-associated cancers in the year 2002. Int J Cancer 2006, 118(12):3030-3044.

13. Marshall BJ, Warren JR: Unidentified curved bacilli in the stomach of patients with gastritis and peptic ulceration. Lancet 1984, 1(8390):1311-1315.

14. de Visser KE, Eichten A, Coussens LM: Paradoxical roles of the immune system during cancer development. Nat Rev Cancer 2006, 6(1):24-37.

15. Viers BR, Boorjian SA, Frank I, Tarrell RF, Thapa P, Karnes RJ, Thompson RH, Tollefson MK:

Pretreatment neutrophil-to-lymphocyte ratio is associated with advanced pathologic tumor stage and increased cancer-specific mortality among patients with urothelial carcinoma of the bladder undergoing radical cystectomy. Eur Uro/ 2014, 66(6):1157-1164.

16. Di Salvo E, Ventura-Spagnolo E, Casciaro M, Navarra M, Gangemi S: IL-33/IL-31 Axis: A Potential Inflammatory Pathway. Mediators Inflamm 2018, 2018:3858032.

17. Singer EM, Shin DB, Nattkemper LA, Benoit BM, Klein RS, Didigu CA, Loren AW, Dentchev T, Wysocka $M$, Yosipovitch $G$ et al: IL-31 is produced by the malignant T-cell population in cutaneous T-Cell lymphoma and correlates with CTCL pruritus. J Invest Dermatol 2013, 133(12):2783-2785.

18. Cedeno-Laurent F, Singer EM, Wysocka M, Benoit BM, Vittorio CC, Kim EJ, Yosipovitch G, Rook AH: Improved pruritus correlates with lower levels of IL-31 in CTCL patients under different therapeutic modalities. Clin Immunol 2015, 158(1):1-7.

19. Joosten LA, Netea MG, Kim SH, Yoon DY, Oppers-Walgreen B, Radstake TR, Barrera P, van de Loo FA, Dinarello CA, van den Berg WB: IL-32, a proinflammatory cytokine in rheumatoid arthritis. Proc NatI Acad Sci U S A 2006, 103(9):3298-3303. 
20. Heinhuis B, Koenders MI, van Riel PL, van de Loo FA, Dinarello CA, Netea MG, van den Berg WB, Joosten LA: Tumour necrosis factor alpha-driven IL-32 expression in rheumatoid arthritis synovial tissue amplifies an inflammatory cascade. Ann Rheum Dis 2011, 70(4):660-667.

21. Marcondes AM, Mhyre AJ, Stirewalt DL, Kim SH, Dinarello CA, Deeg HJ: Dysregulation of IL-32 in myelodysplastic syndrome and chronic myelomonocytic leukemia modulates apoptosis and impairs NK function. Proc Natl Acad Sci U S A 2008, 105(8):2865-2870.

22. Oh JH, Cho MC, Kim JH, Lee SY, Kim HJ, Park ES, Ban JO, Kang JW, Lee DH, Shim JH et al: IL-32Y inhibits cancer cell growth through inactivation of NF-KB and STAT3 signals. Oncogene 2011, 30(30):3345-3359.

23. Nishida A, Andoh A, Inatomi O, Fujiyama Y: Interleukin-32 expression in the pancreas. J Biol Chem 2009, 284(26):17868-17876.

24. Sakitani K, Hirata Y, Hayakawa Y, Serizawa T, Nakata W, Takahashi R, Kinoshita H, Sakamoto K, Nakagawa $\mathrm{H}$, Akanuma $\mathrm{M}$ et al: Role of interleukin-32 in Helicobacter pylori-induced gastric inflammation. Infect Immun 2012, 80(11):3795-3803.

25. Gonzalez-Hormazabal P, Musleh M, Bustamante M, Stambuk J, Escandar S, Valladares H, Lanzarini $\mathrm{E}$, Chiong $\mathrm{H}$, Rojas J, Castro VG et al: Role of cytokine gene polymorphisms in gastric cancer risk in Chile. Anticancer Res 2014, 34(7):3523-3530.

26. Hu LA, Fu Y, Zhang DN, Zhang J: Serum IL-33 as a diagnostic and prognostic marker in non- small cell lung cancer. Asian Pac J Cancer Prev 2013, 14(4):2563-2566.

27. Naumnik W, Naumnik B, Niewiarowska K, Ossolinska M, Chyczewska E: Novel cytokines: IL-27, IL-29, IL-31 and IL-33. Can they be useful in clinical practice at the time diagnosis of lung cancer? Exp Oncol 2012, 34(4):348-353.

28. Liu QH, Shi ML, Bai J, Zheng JN: Identification of ANXA1 as a lymphatic metastasis and poor prognostic factor in pancreatic ductal adenocarcinoma. Asian Pac J Cancer Prev 2015, 16(7):27192724.

29. Chen F, Qu M, Zhang F, Tan Z, Xia Q, Hambly BD, Bao S, Tao K: IL-36 s in the colorectal cancer: is interleukin 36 good or bad for the development of colorectal cancer? BMC Cancer 2020, 20(1):92.

30. Liu Q, Zhang Y, Zhang J, Tao K, Hambly BD, Bao S: Inverse correlation between Interleukin-34 and gastric cancer, a potential biomarker for prognosis. Cell Biosci 2020, 10:94.

31. Nakamura T, Yao T, Niho Y, Tsuneyoshi M: A clinicopathological study in young patients with gastric carcinoma. J Surg Oncol 1999, 71(4):214-219.

32. Diveu C, Lelievre E, Perret D, Lak-Hal AH, Froger J, Guillet C, Chevalier S, Rousseau F, Wesa A, Preisser $\mathrm{L}$ et al: GPL, a novel cytokine receptor related to GP130 and leukemia inhibitory factor receptor. $J$ Biol Chem 2003, 278(50):49850-49859.

33. Zhang Q, Putheti P, Zhou Q, Liu Q, Gao W: Structures and biological functions of IL-31 and IL-31 receptors. Cytokine Growth Factor Rev 2008, 19(5-6):347-356.

34. Diveu C, Lak-Hal AH, Froger J, Ravon E, Grimaud L, Barbier F, Hermann J, Gascan H, Chevalier S: Predominant expression of the long isoform of GP130-like (GPL) receptor is required for interleukin- 
31 signaling. Eur Cytokine Netw 2004, 15(4):291-302.

35. Ferretti E, Tripodo C, Pagnan G, Guarnotta C, Marimpietri D, Corrias MV, Ribatti D, Zupo S, FraternaliOrcioni G, Ravetti JL et al: The interleukin (IL)-31/IL-31R axis contributes to tumor growth in human follicular lymphoma. Leukemia 2015, 29(4):958-967.

36. Pyo JH, Lee H, Min BH, Lee JH, Choi MG, Lee JH, Sohn TS, Bae JM, Kim KM, Ahn HS et al: A Risk Prediction Model Based on Lymph-Node Metastasis in Poorly Differentiated-Type Intramucosal Gastric Cancer. PLoS One 2016, 11(5):e0156207.

37. Bonilla WV, Frohlich A, Senn K, Kallert S, Fernandez M, Johnson S, Kreutzfeldt M, Hegazy AN, Schrick $C$, Fallon $\mathrm{PG}$ et al: The alarmin interleukin-33 drives protective antiviral CD8(+) T cell responses. Science 2012, 335(6071):984-989.

38. Liew FY, Pitman NI, Mclnnes IB: Disease-associated functions of IL-33: the new kid in the IL-1 family. Nat Rev Immuno/ 2010, 10(2):103-110.

39. Chackerian AA, Oldham ER, Murphy EE, Schmitz J, Pflanz S, Kastelein RA: IL-1 receptor accessory protein and ST2 comprise the IL-33 receptor complex. J Immunol 2007, 179(4):2551-2555.

40. Hayakawa $H$, Hayakawa $M$, Kume A, Tominaga S: Soluble ST2 blocks interleukin-33 signaling in allergic airway inflammation. J Biol Chem 2007, 282(36):26369-26380.

41. Gao K, Li X, Zhang L, Bai L, Dong W, Gao K, Shi G, Xia X, Wu L, Zhang L: Transgenic expression of IL33 activates $\operatorname{CD} 8(+)$ T cells and NK cells and inhibits tumor growth and metastasis in mice. Cancer Lett 2013, 335(2):463-471.

42. Ishigami S, Arigami T, Uchikado Y, Setoyama T, Kita Y, Sasaki K, Okumura H, Kurahara H, Kijima Y, Harada A et al: IL-32 expression is an independent prognostic marker for gastric cancer. Med Oncol 2013, 30(2):472.

43. Nabeki B, Ishigami S, Uchikado Y, Sasaki K, Kita Y, Okumura H, Arigami T, Kijima Y, Kurahara H, Maemura $\mathrm{K}$ et al: Interleukin-32 expression and Treg infiltration in esophageal squamous cell carcinoma. Anticancer Res 2015, 35(5):2941-2947.

44. Netea MG, Lewis EC, Azam T, Joosten LA, Jaekal J, Bae SY, Dinarello CA, Kim SH: Interleukin-32 induces the differentiation of monocytes into macrophage-like cells. Proc Natl Acad Sci U S A 2008, 105(9):3515-3520.

45. Ley K: M1 Means Kill; M2 Means Heal. J Immunol 2017, 199(7):2191-2193.

46. Bao S, Hu R, Hambly BD: IL-34, IL-36 and IL-38 in colorectal cancer-key immunoregulators of carcinogenesis. Biophys Rev 2020, 12(4):925-930.

47. Heinhuis B, Plantinga TS, Semango G, Kusters B, Netea MG, Dinarello CA, Smit JWA, Netea-Maier RT, Joosten LAB: Alternatively spliced isoforms of IL-32 differentially influence cell death pathways in cancer cell lines. Carcinogenesis 2016, 37(2):197-205.

48. Yun HM, Oh JH, Shim JH, Ban JO, Park KR, Kim JH, Lee DH, Kang JW, Park YH, Yu D et al: Antitumor activity of IL-32beta through the activation of lymphocytes, and the inactivation of NF-kappaB and STAT3 signals. Cell Death Dis 2013, 4:e640. 
49. Meyer N, Christoph J, Makrinioti H, Indermitte P, Rhyner C, Soyka M, Eiwegger T, Chalubinski M, Wanke K, Fujita $\mathrm{H}$ et al: Inhibition of angiogenesis by IL-32: possible role in asthma. J Allergy Clin Immunol 2012, 129(4):964-973 e967.

\section{Tables}

Due to technical limitations, table 1, table 2 and table 3 are only available as a download in the Supplemental Files section.

\section{Figures}

Figure 1

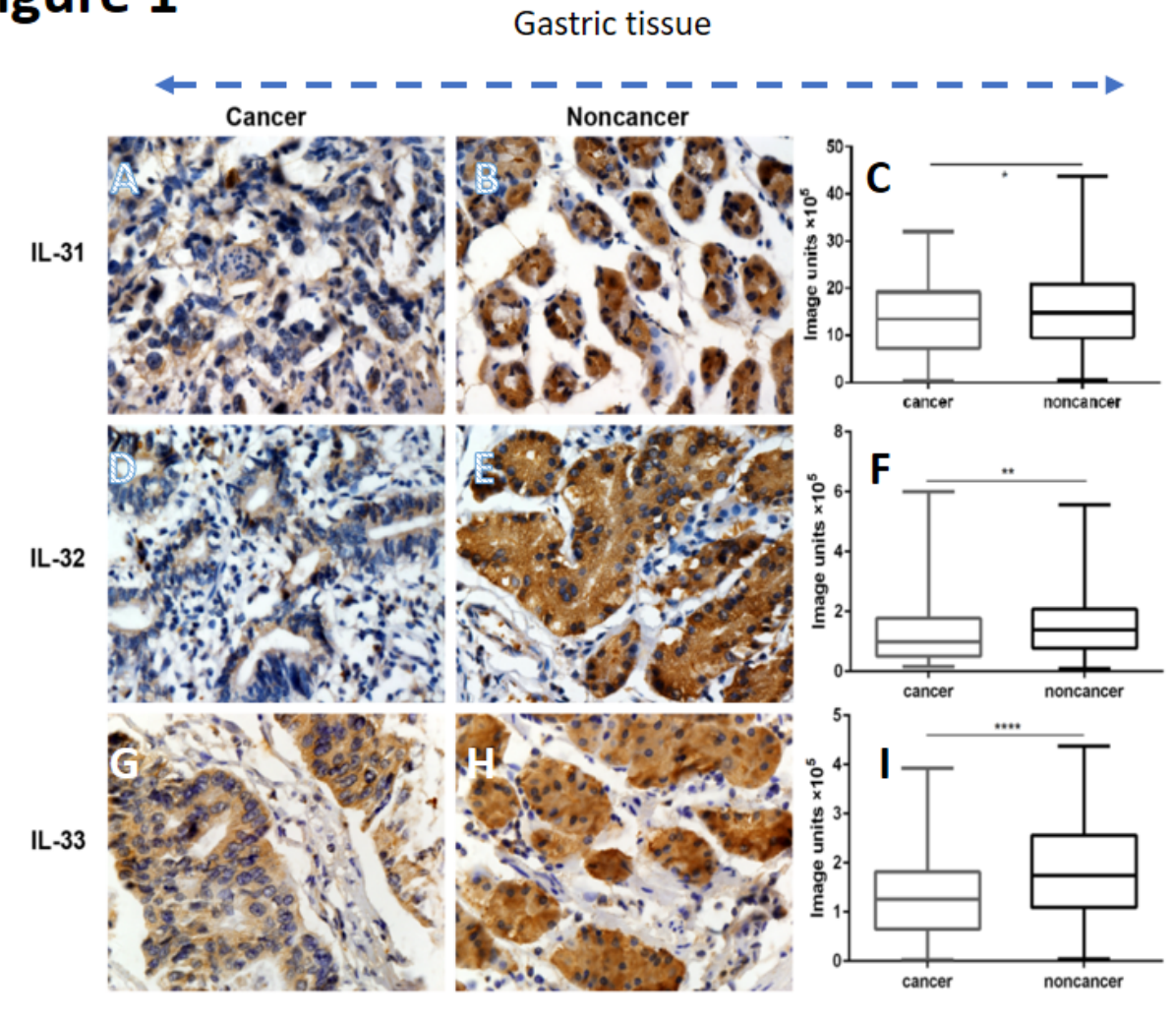

Peripheral blood
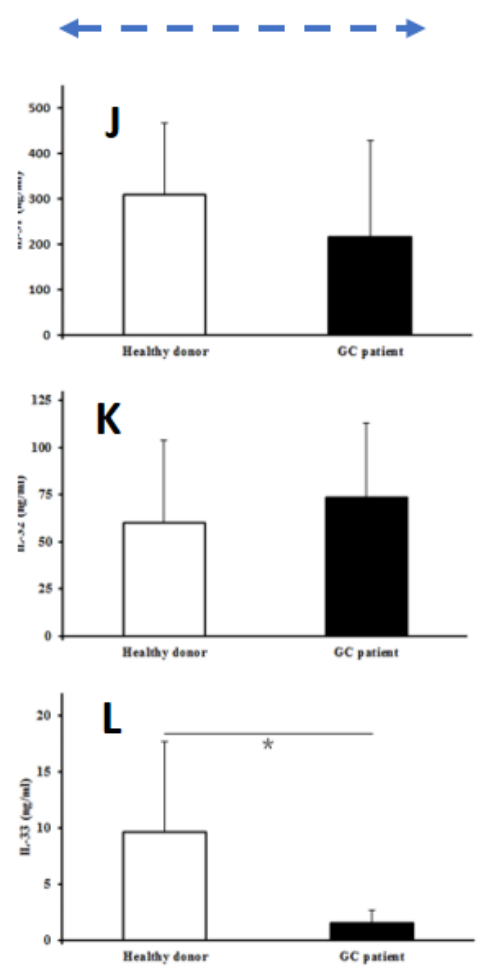

\section{Figure 1}

Representative images of IL-31, IL-32 and IL-33 immunohistochemical stained and their densities in noncancerous and GC tissues, as well IL-31, IL-32 and IL-33 levels in peripheral blood of GC patients and healthy individuals Positive (brown) IL-31 in the cytoplasm of gastric cancer (A) and noncancerous tissues (B) and the quantified data (C). Positive IL-32 in the cytoplasm of gastric cancer (D) and noncancerous tissues (E) and the quantified data (F). Positive IL-33 in the cytoplasm of gastric cancer (G) and noncancerous tissues $(\mathrm{H})$ and the quantified data (I). The densities of IL-31, and IL-33 were all decreased in GC compared to tumour adjacent normal gastric tissues. Magnification, $600 \times$ IL-31 (J), 32 $(\mathrm{K}), 33(\mathrm{~L})$ levels in the peripheral blood from GC patients and healthy control was displayed, as well. 


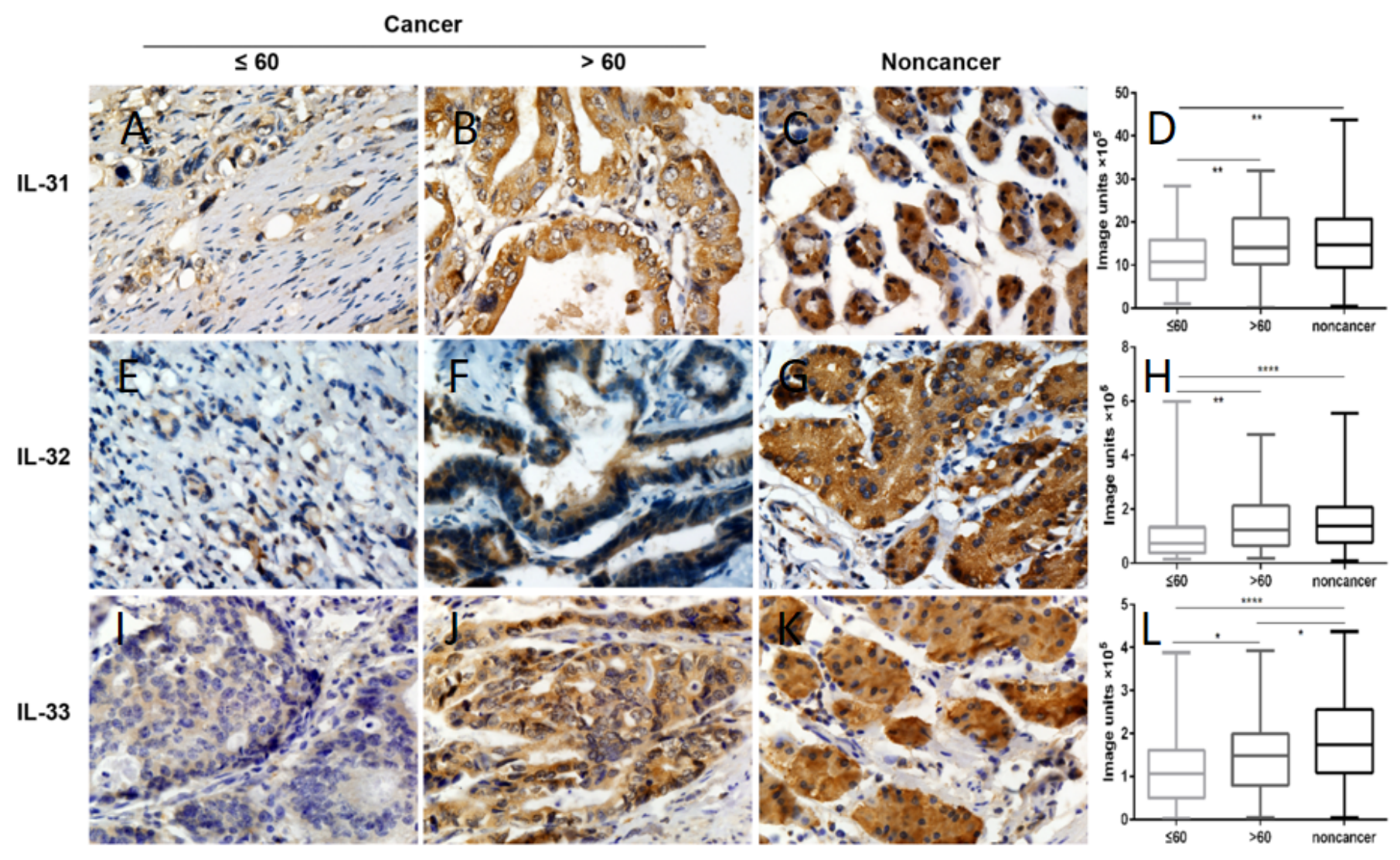

Figure 2

Correlation of IL-31, IL-32 and IL-33 expressions with age subgroups Positive (brown) IL-31 in the cytoplasm of gastric cancer $<60$ (A) vs $>60$ (B), and noncancerous tissues (C) and the quantified data (D). Positive (brown) IL-32 in the cytoplasm of gastric cancer $<60$ (E) vs $>60$ (F), and noncancerous tissues (G) and the quantified data (H). Positive (brown) IL-33 in the cytoplasm of gastric cancer $<60$ (I) vs >60 $(\mathrm{J})$, and noncancerous tissues (K) and the quantified data (L). IL-32 and IL-33 all decreased in the group of GC patients aged less than or equal to 60 years. 


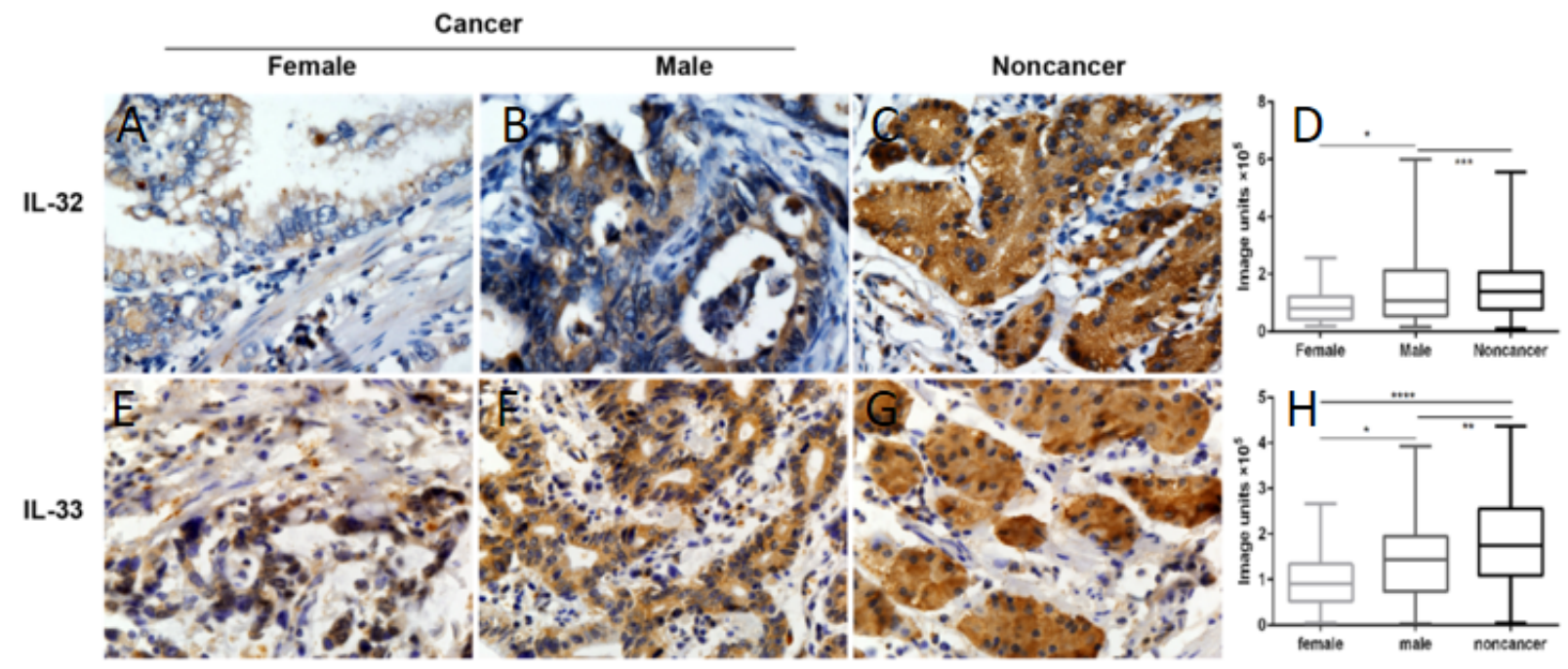

\section{Figure 3}

Correlation of IL-32 and IL-33 expressions with gender subtype Positive (brown) IL-32 in the cytoplasm of gastric cancer female (A) vs male (B), and noncancerous tissues (C) and the quantified data (D). Positive (brown) IL-33 in the cytoplasm of gastric cancer female (E) vs $>60(F)$, and noncancerous tissues (G) and the quantified data $(\mathrm{H}) . \mathrm{IL}-32$ and IL-33 both decreased in the female subgroup patients with GC. 
IL-31

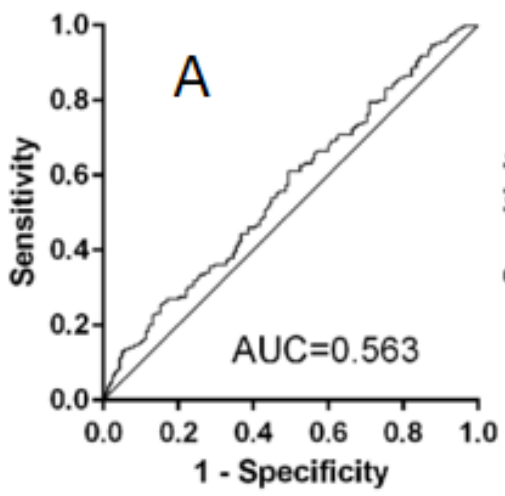

IL-31

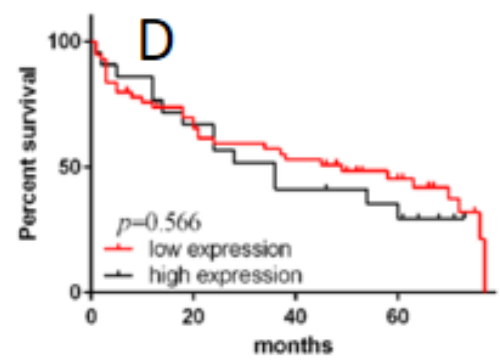

IL-31 in T4 tumor invasion depth

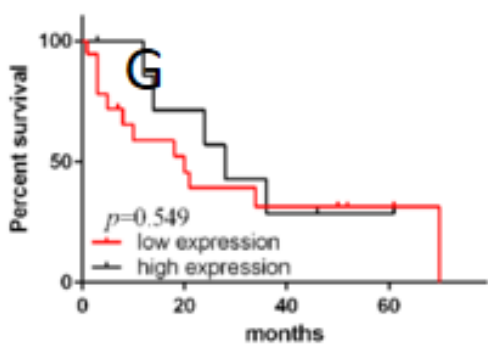

IL-31 in TNM I-II

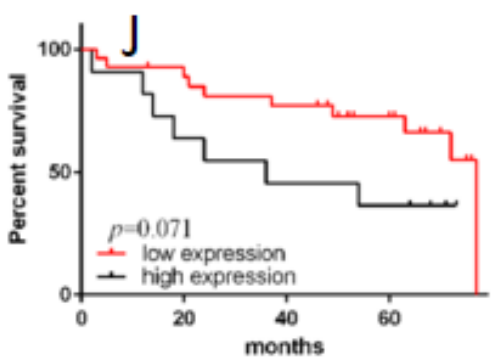

IL-32

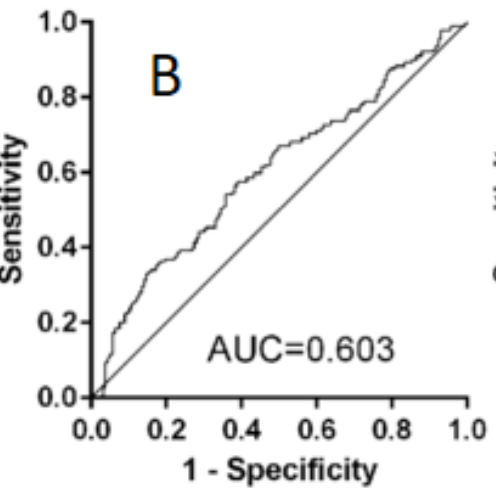

IL-32

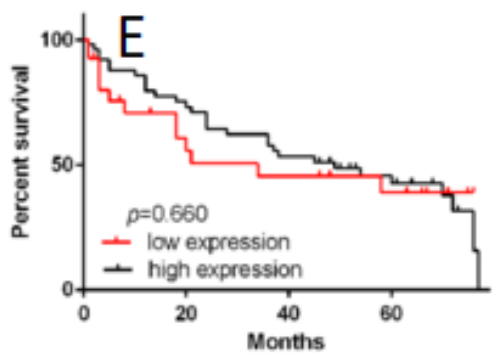

IL-32 in T4 tumour invasion depth

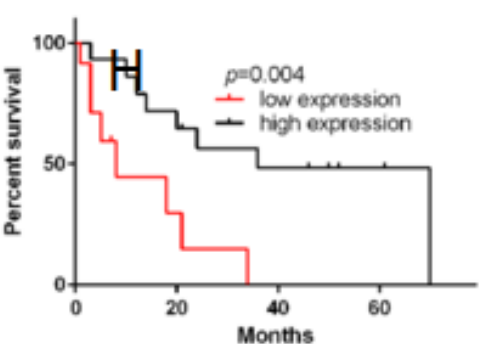

IL-32 in TNM I-II

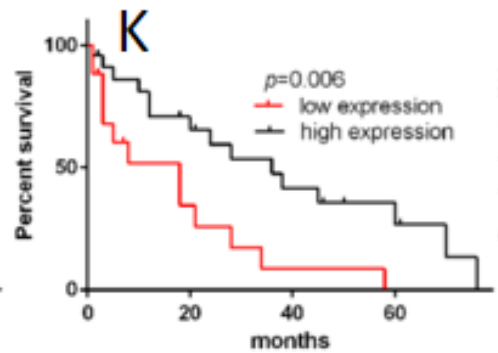

IL-33

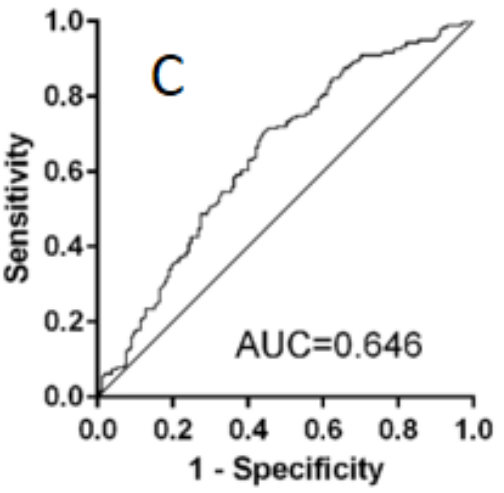

IL-33

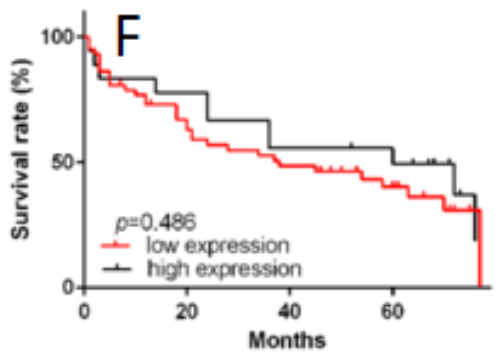

IL-33 in T4 tumour invasion depth

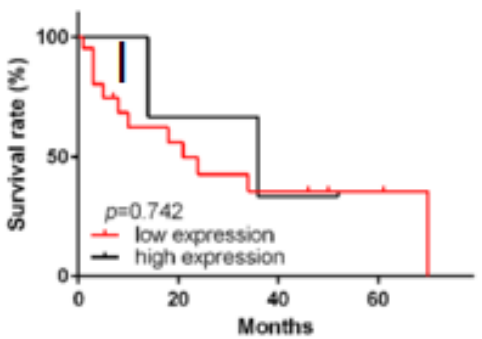

IL-33 in TNM I-II

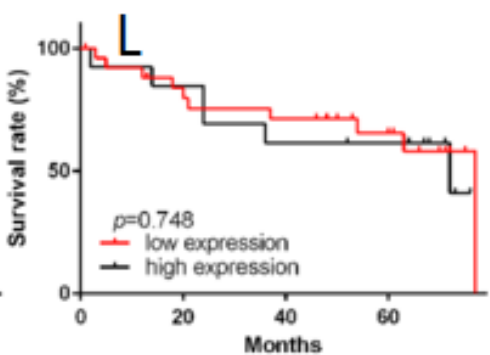

\section{Figure 4}

ROC curves and correlation of IL-31, IL-32 and IL-33 for prognosis of GC, plus subgroup analysis for tumour invasion depth T4 patients and TNM I-II patients with GC The specificity (X-axis) vs sensitivity (Yaxis) of IL-31 (A), IL-32 (B) and IL-33 (C) are presented. Comparison of 5 year survival rate between high and low IL-31 (D), IL-32 (E), IL-33 (F) are presented among the whole GC patients in the study. Comparison of 5 year survival rate between high and low IL-31 (G), IL-32 (H), IL-33 (I) are presented 
among the GC patients in T4 depth invasion. Comparison of 5 year survival rate between high and low IL$31(\mathrm{~J}), \mathrm{IL}-32(\mathrm{~K}), \mathrm{IL}-33(\mathrm{~L})$ are presented among the GC patients in TNM stage 2. ROC curves analysis displaying the poor diagnostic potential for GC by the expression levels of IL-31, IL-32 and IL-33. The cutoff point and area under the curve were: IL-31: 1.486×106, AUC=0.563; IL-32: 64893, AUC=0.603; IL-33: 166291, AUC $=0.646$. Kaplan-Meier survival analysis of GC patients: Decreased IL-32 expression correlates with poor survival of GC patients within the tumour invasion depth T4 and TNM I-II subgroups of GC.

\section{Supplementary Files}

This is a list of supplementary files associated with this preprint. Click to download.

- Table1.pdf

- Table2.pdf

- Table3.pdf

- supplementaryfigures.pdf 